Supporting information

\title{
Hyaluronic Acid-Decorated Glycol Chitosan Nanoparticles for pH-Sensitive Controlled Release of Doxorubicin and Celecoxib in Nonsmall Cell Lung Cancer
}

Ruda Lee 1 , Yu Jin Choi ${ }^{2}$, Myeong Seon Jeong ${ }^{2}$, Yong Il Park ${ }^{3}$, Keiichi Motoyama ${ }^{4}$, Min Woo $\mathrm{Kim}^{1}$, Seung-Hae Kwon ${ }^{5, *}$ and Jung Hoon Choi ${ }^{6, *}$

\footnotetext{
${ }^{1}$ International Research of Advanced Science and Technology, Kumamoto University, Kumamoto 860-8555, Japan

${ }^{2}$ Korea Basic Science Institute, Chuncheon 24341, Republic of Korea

${ }^{3}$ School of Chemical Engineering, Chonnam National University, Gwangju 61186, Republic of Korea

${ }^{4}$ Graduate School of Pharmaceutical Sciences, Kumamoto University, Kumamoto 860-8555, Japan

${ }^{5}$ Korea Basic Science Institute, Seoul 02841, Republic of Korea

${ }^{6}$ Department of Anatomy \& Institute of Veterinary Science, College of Veterinary Medicine, Kangwon National University, Chuncheon 24341, Republic of Korea
}

Supplement Figure Contents $(1-7)$ 


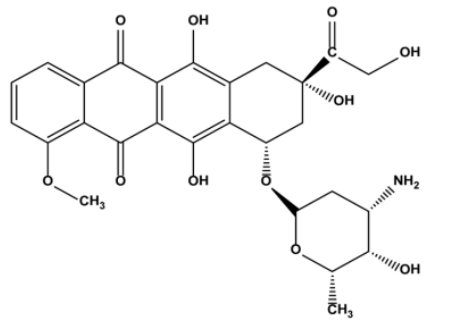

Doxolubicin $\cdot \mathrm{HCl}(\mathrm{DOX})$

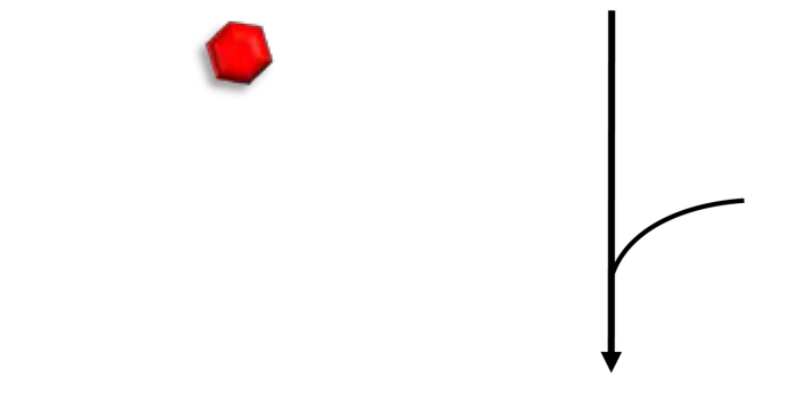

$+$
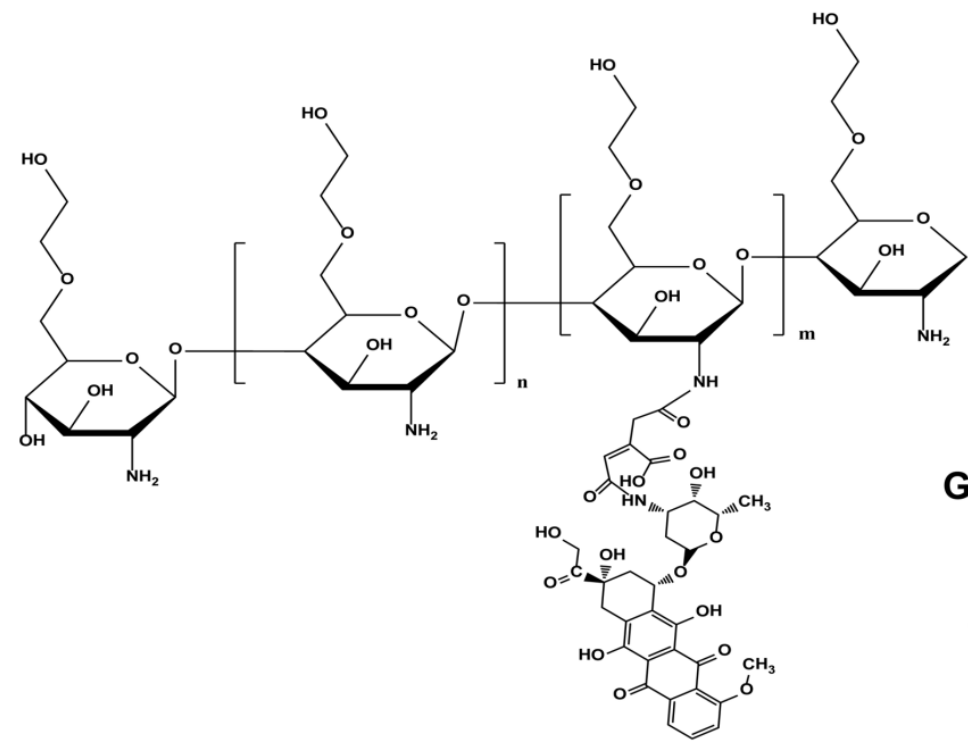

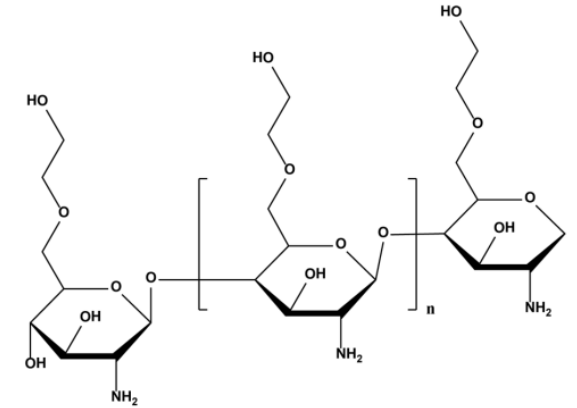

Glycol chitosan (GC)

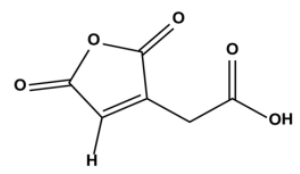

cis-Aconitic anhydride (cA)

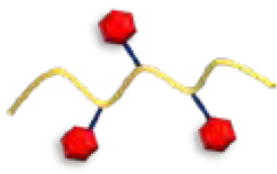

Figure S1. Chemical structure of GC-DOX. DOX was conjugated to GC via EDC-NHS reaction using a $\mathrm{pH}$-sensitive (cA) linker. 


\section{A}

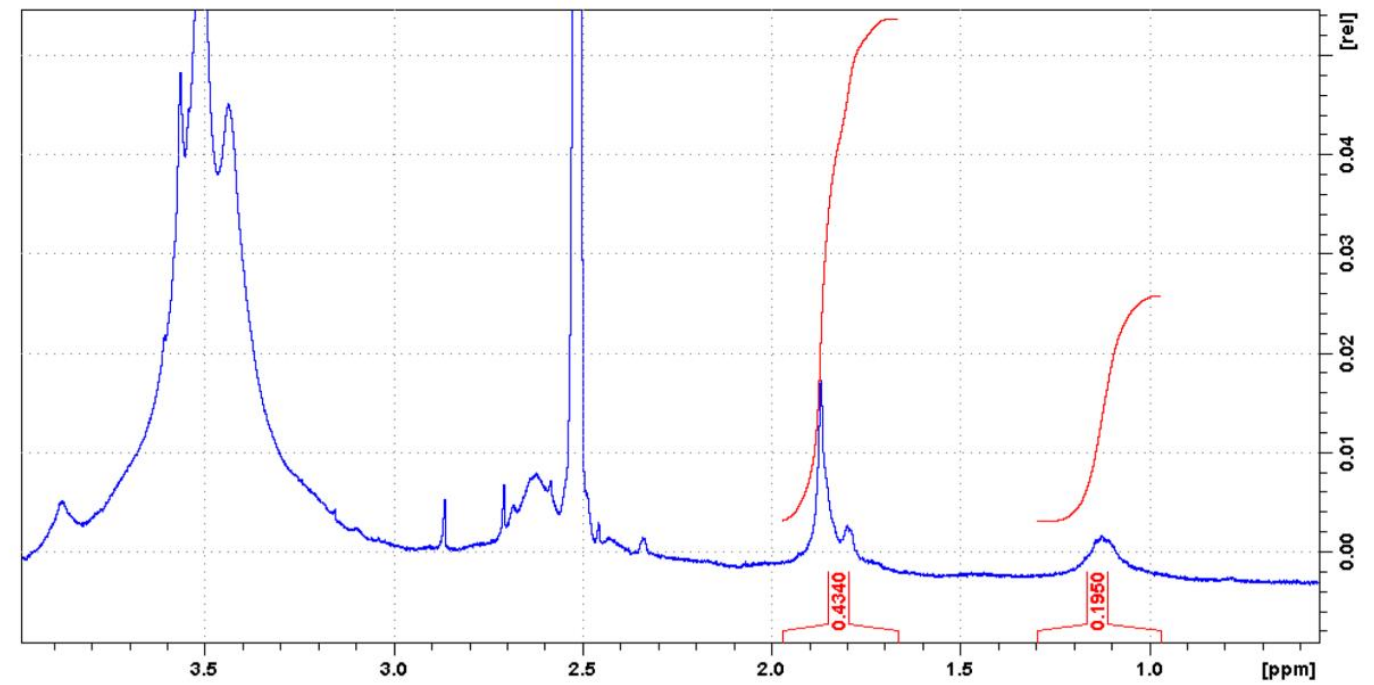

B

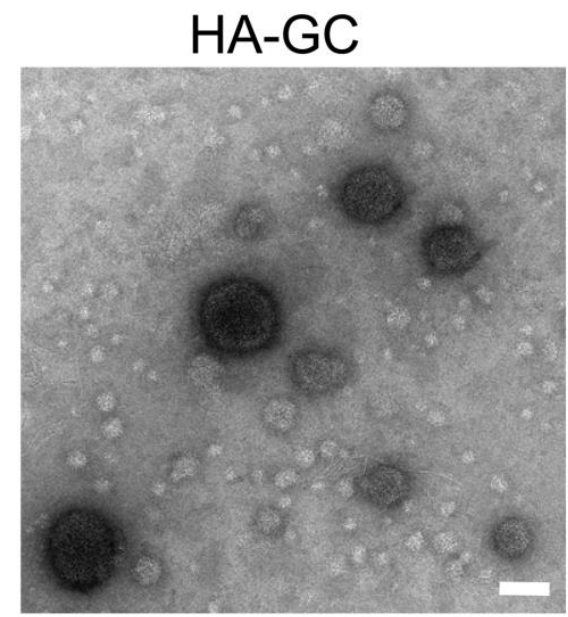

C

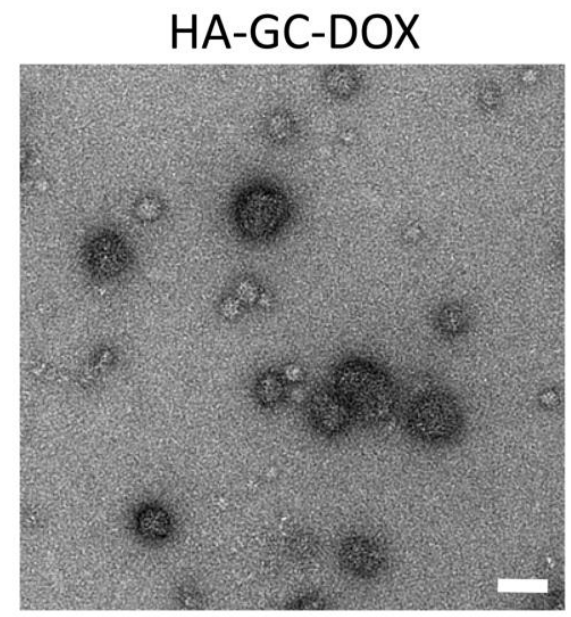

Figure S2. Quantitative analysis of DOX conjugation and TEM images of HA-GC and HA-GC-DOX. A. Quantitative NMR analysis of DOX conjugated GC. Three different GC-DOX batches were analyzed.

B. Representative TEM images of HA-GC, C. Representative TEM images of HA-GC-DOX $(n=5)$. Scale bar $=100 \mathrm{~nm}$ 


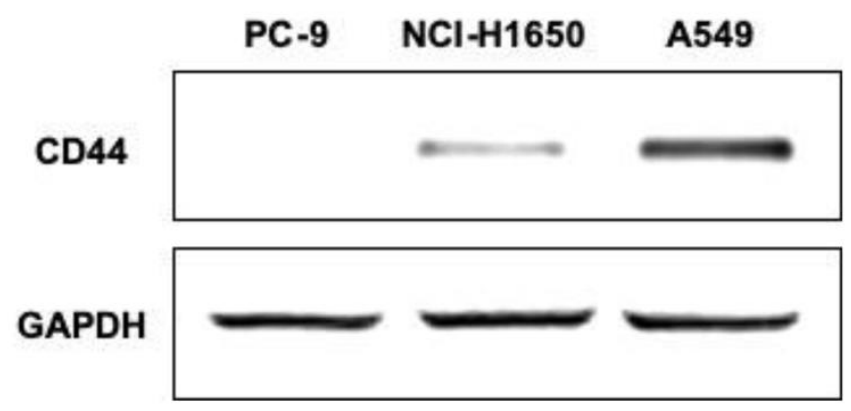

Figure S3. Cellular level of CD44 in three different NSCLC. GAPDH was used as an internal control ( $n$ $=3)$. 


\section{A}

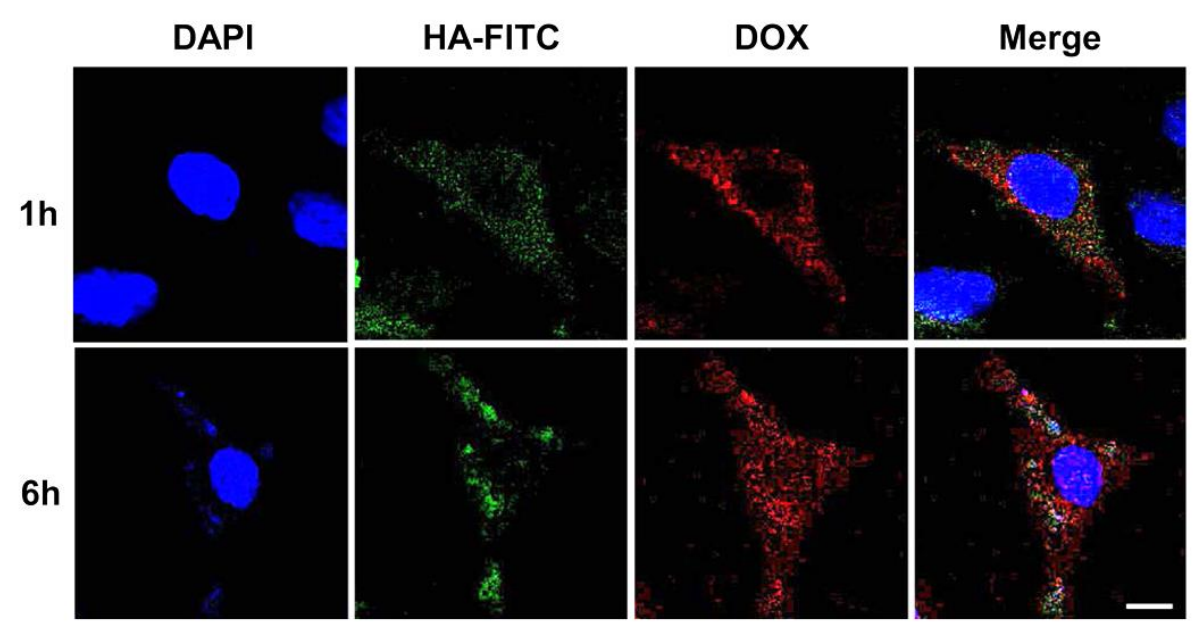

B

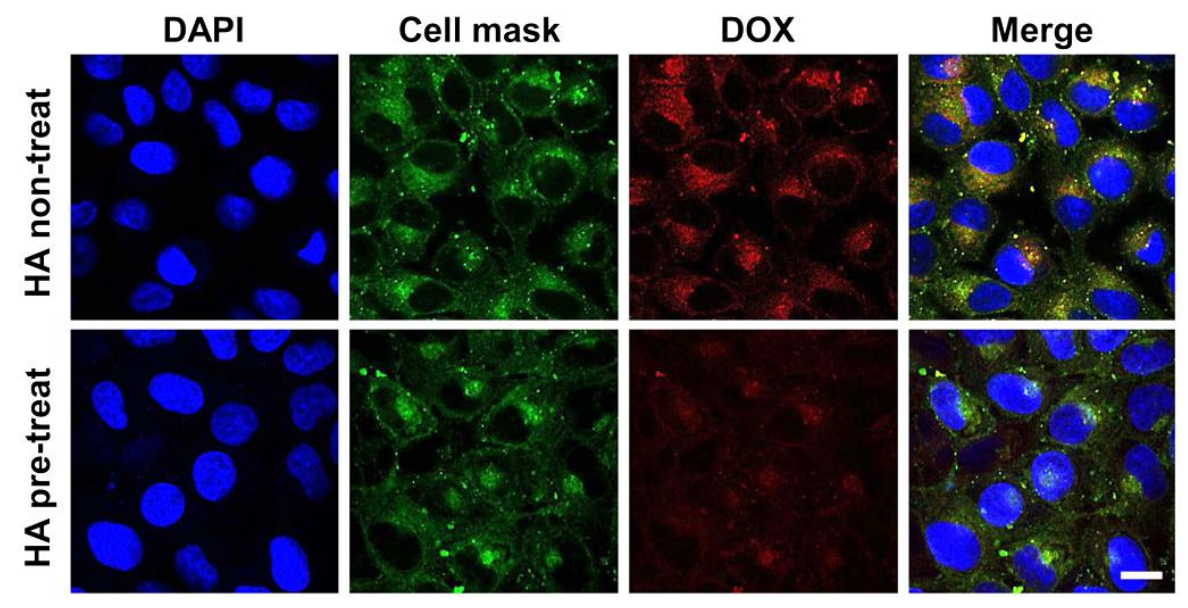

Figure S4. In vitro cellular drug release and endocytosis pathway of HA-GC-DOX/CXB. A. Representative images of DOX released from HA(FITC)-GC-DOX/CXB. A549-Luc cells were incubated with HA-GC-DOX/CXB for $1 \mathrm{~h}$ and $6 \mathrm{~h}$. B. Representative images of A549-Luc cells incubated with HA-GC-DOX/CXB under pre-treated or non-treated HA $(2 \mathrm{mg} / \mathrm{mL})$. Scale bar $=20 \mu \mathrm{m}$. 
A

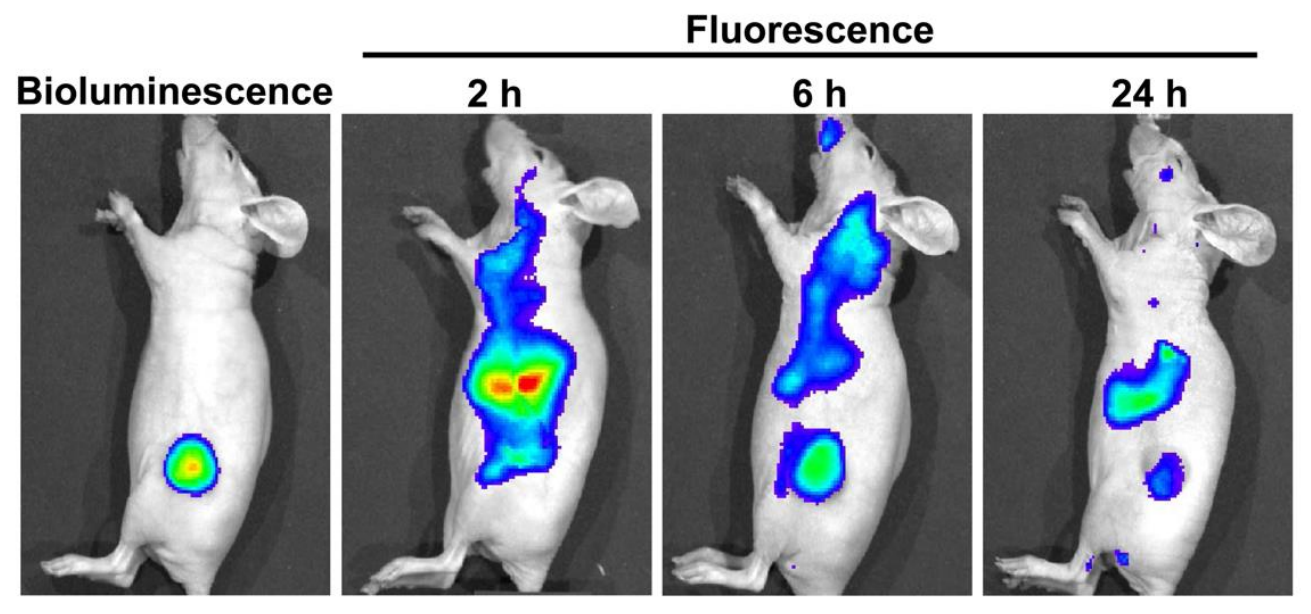

B
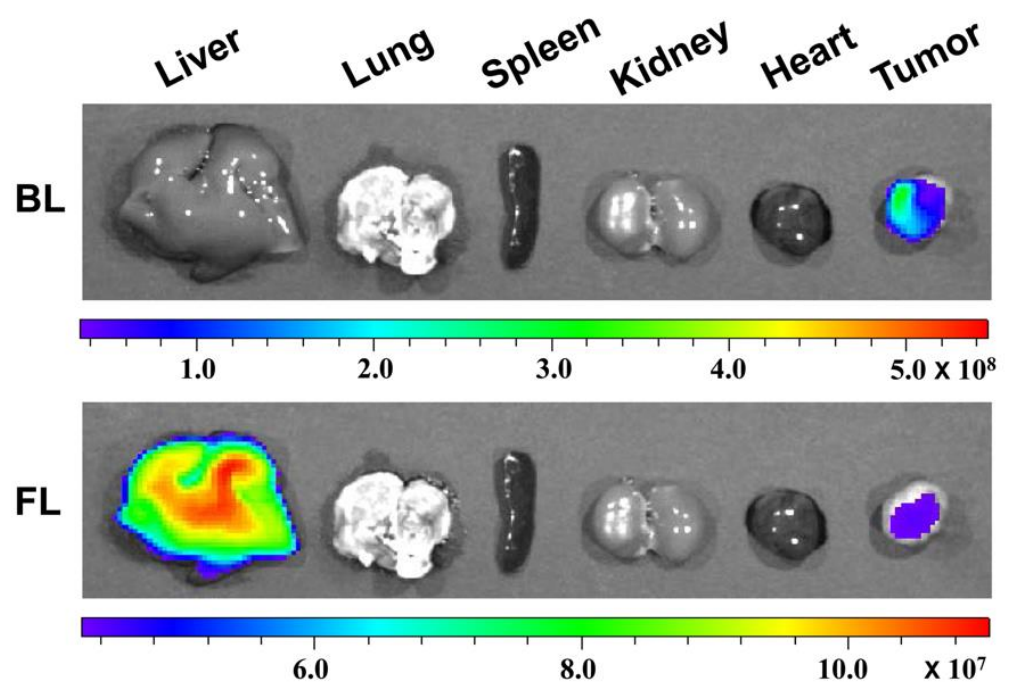

Figure S5. Biodistribution of HA-GC-Cy5.5 NPs. A. In vivo biodistribution of HA-GC-Cy5.5 NPs in A549-Luc tumor mice. Representative luminescence and NIR fluorescence images were obtained at 2, 6, and $24 \mathrm{~h}$ after administered HA-GC-Cy5.5 NPs $(100 \mu \mathrm{g}, n=3)$. B. Ex vivo dissected organ images were obtained at $24 \mathrm{~h}$ post injected HA-GC-Cy5.5 NPs $(n=3)$. 


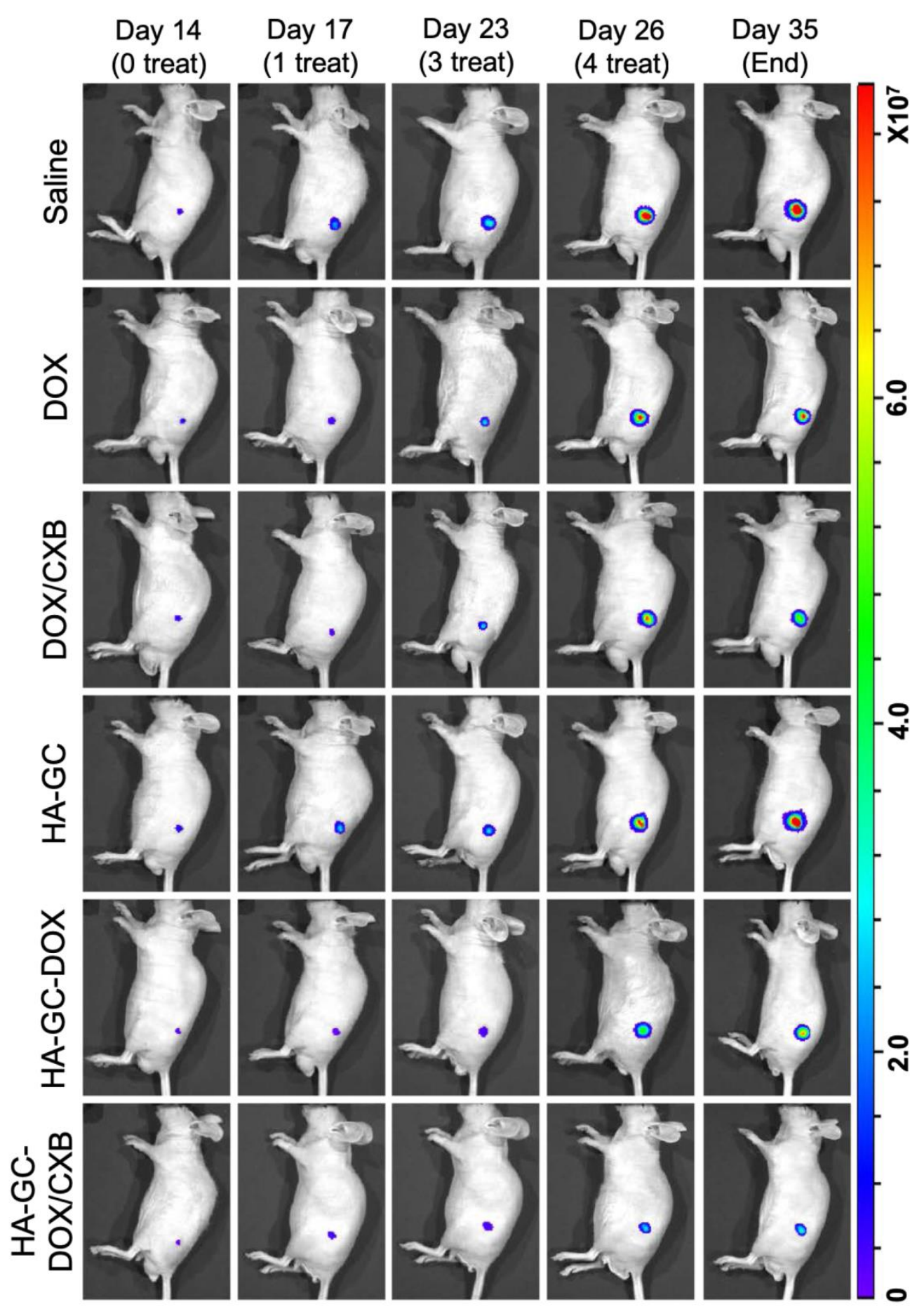

Figure S6. Evaluation of in vivo bioluminescence signals at 14, 17, 23, 26, and 35 days post A549-Luc cells inoculation, that is, at baseline and after $1^{\text {st }}, 3^{\text {rd }}$, and $4^{\text {th }}$ treatments, respectively $(n=5)$. 


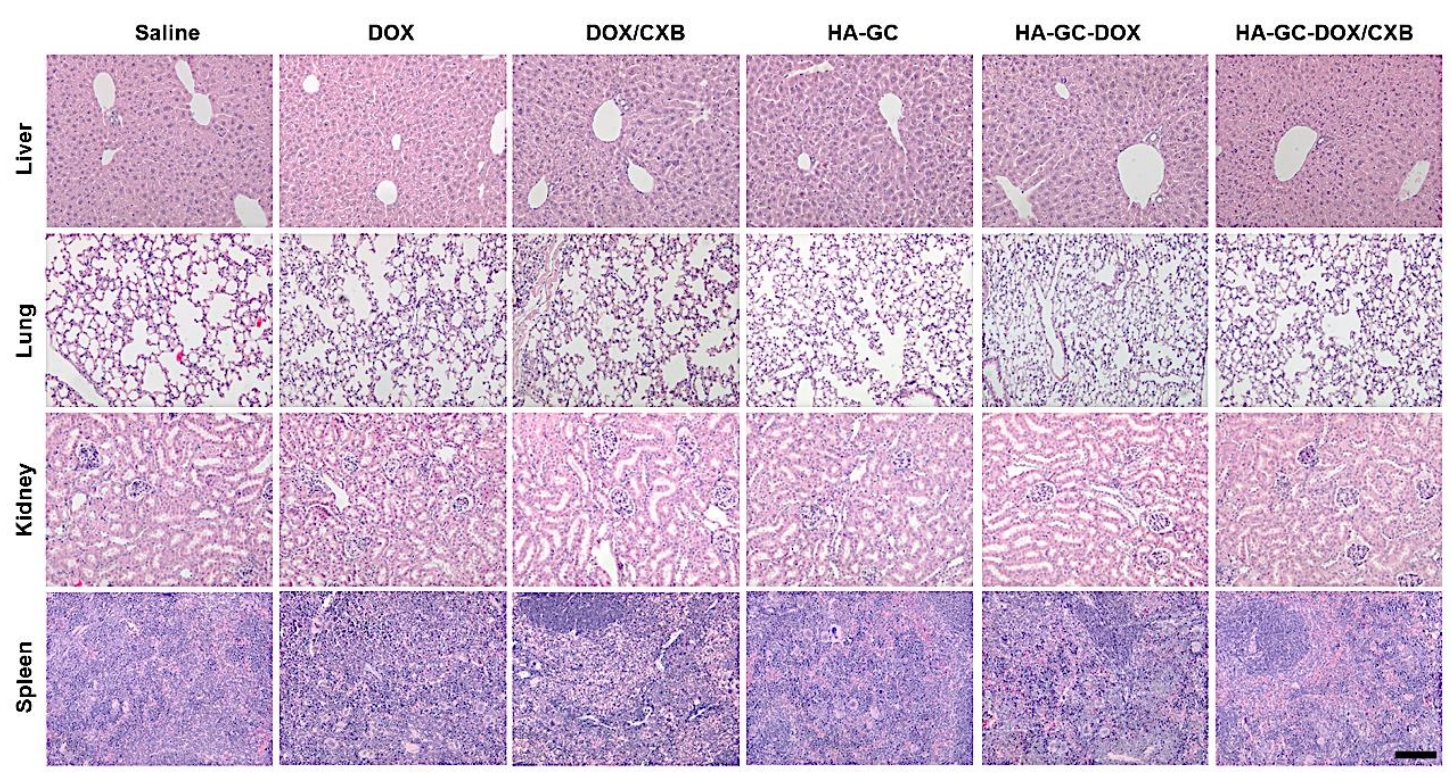

Figure S7. Histological analysis of organ toxicity $(n=3)$ by hematoxylin $\&$ eosin staining. Scale bar $=$ $100 \mu \mathrm{m}$. 